\title{
Tertiary peritonitis: considerations for complex team-based care
}

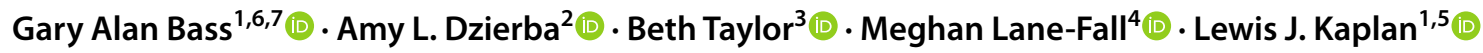

Received: 4 May 2021 / Accepted: 18 July 2021 / Published online: 24 July 2021

(c) Springer-Verlag GmbH Germany, part of Springer Nature 2021

\begin{abstract}
Peritonitis, as a major consequence of hollow visceral perforation, anastomotic disruption, ischemic necrosis, or other injuries of the gastrointestinal tract, often drives acute care in the emergency department, operating room, and the ICU. Chronic critical illness (CCI) represents a devastating challenge in modern surgical critical care where successful interventions have fostered a growing cohort of patients with prolonged dependence on mechanical ventilation and other organ supportive therapies who would previously have succumbed much earlier in the acute phase of critical illness. An important subset of CCI patients are those who have survived an emergency abdominal operation, but who subsequently require prolonged open abdomen management complicated by persistent peritoneal space infection or colonization, fistula formation, and gastrointestinal (GI) tract dysfunction; these patients are described as having tertiary peritonitis (TP). The organ dysfunction cascade in TP terminates in death in between 30 and $64 \%$ of patients. This narrative review describes key—but not all-elements in a framework for the coordinate multiprofessional team-based management of a patient with tertiary peritonitis to mitigate this risk of death and promote recovery. Given the prolonged critical illness course of this unique patient population, early and recurrent Palliative Care Medicine consultation helps establish goals of care, support adjustment to changes in life circumstance, and enable patient and family centered care.
\end{abstract}

Keywords Tertiary peritonitis $\cdot$ Surgery $\cdot$ Critical care $\cdot$ Mortality $\cdot$ Infection $\cdot$ Morbidity

\section{Abbreviations}

ACS Abdominal compartment syndrome

ACTH Adrenocorticotrophic hormone (corticotropin)

AKI Acute kidney injury

Presented as an Invited Lecture to the virtual European Society of Trauma and Emergency Surgery (ESTES) Week, April 25-30th 2021.

Gary Alan Bass

gary.bass@pennmedicine.upenn.edu

Amy L. Dzierba

ald9012@nyp.org

Beth Taylor

beth.taylor@bjc.org

Meghan Lane-Fall

Meghan.lanefall@pennmedicine.upenn.edu

Lewis J. Kaplan

lewis.kaplan@pennmedicine.upenn.edu;

Lewis.Kaplan@va.gov

1 Division of Traumatology, Surgical Critical Care and Emergency Surgery, Perelman School of Medicine, University of Pennsylvania, 51 N. 39th Street, MOB 1, Suite 120, Philadelphia, PA 19104, USA
ARDS Acute respiratory distress syndrome

BCAA Branch-chain amino acid

CCI Chronic critical illness

EAF Enteroatmospheric fistula

ECF Enterocutaneous fistula
2 Department of Pharmacy, New York-Presbyterian Hospital, Columbia University Irving Medical Center, New York, NY, USA

3 Department of Research for Patient Care Services, Barnes-Jewish Hospital, St. Louis, MO, USA

4 Department of Anesthesia and Critical Care, Perelman School of Medicine, University of Pennsylvania, 3400 Spruce Street, 5 Dulles, Philadelphia, PA 19104, USA

5 Surgical Services, Section of Surgical Critical Care, Corporal Michael J Crescenz VA Medical Center, 3900 Woodland Avenue, Philadelphia, PA 19104, USA

6 Leonard Davis Institute of Health Economics, University of Pennsylvania, Philadelphia, USA

7 European Society of Trauma and Emergency Surgery, Visceral Trauma Section, Philadelphia, USA 


$\begin{array}{ll}\text { FA } & \text { Fatty acids } \\ \text { GH } & \text { Growth hormone } \\ \text { HMB } & \beta \text {-Hydroxy } \beta \text {-methylbutyrate } \\ \text { ICU } & \text { Intensive care unit } \\ \text { IGF } & \text { Insulin-like growth hormone } \\ \text { MDRO } & \text { Multidrugresistant organisms } \\ \text { mTOR } & \text { Mammalian target of rapamycin } \\ \text { NPWT } & \text { Negative pressure wound therapy } \\ \text { OR } & \text { Operating room } \\ \text { SPM } & \text { Specialized pre-resolving mediators } \\ \text { TP } & \text { Tertiary peritonitis } \\ \text { TSH } & \text { Thyroid stimulating hormone (thyrotropin) }\end{array}$

\section{Introduction}

Peritonitis, as a major consequence of hollow visceral perforation, anastomotic disruption, ischemic necrosis, or other injuries of the gastrointestinal tract, often drives acute care in the emergency department, operating room, and the ICU [1-3]. Resuscitation coupled with rapid source control of the infectious focus and appropriate empiric antibiotic therapy to reduce the local and remote bacterial bioburden underpins effective treatment [4-7]. Such an integrated approach is embraced by a variety of medical professional organization guidelines as well as the Surviving Sepsis Campaign [8]. Concomitant SARS-CoV-2 infection augments clinician safety precautions but does not alter the key tenets of peritonitis management articulated above [9]. The outcomes of acute peritonitis management depend on etiology, chronic comorbidities, time of recognition, and unique aspects of genotype and phenotype that are being explored in terms of immune competency and inflammatory profiling that relate to sepsis $[10,11]$. While many rapidly recover, and some are unable to be salvaged when presenting in extremis, others may demonstrate a long hospital course marked by prolonged ICU length of stay, repeated infection, persistent inflammation, hypercatabolism, and non-resolving organ failure. This constellation of findings represents an untoward outcome termed chronic critical illness-a syndrome that is not limited to patients with surgical disease, but has been best investigated in surgical populations [12].

\section{Methods}

We conducted a narrative review of the literature, using broad search criteria, to provide practical guidance around key management aspects important in the care of the patient with tertiary peritonitis. Following the SANRA guidelines for narrative reviews [13], we aim to summarize pertinent literature in a way which is not explicitly systematic but instead frames some of the complexities around tertiary peritonitis for practicing acute care surgeons, and explore what is and what is not known [14]. To that end, OVID and PubMed databases (supplemented by secondary reference material) were queried for English language manuscripts regarding tertiary peritonitis from inception through March 1,2021 . Focused domains included surgical therapy, critical care management, acute kidney injury, organ failure, host defense, nutritional support, pharmacodynamics and pharmacokinetics, chronic critical illness, as well as operating room and procedural sedation, analgesia, and anesthesia. Primary data, reviews, systematic reviews, and meta-analyses were considered addressing human data as well as animal, cell, or molecular focused inquiries that addressed mechanisms. Included manuscripts were selected as they related to complex care and especially team-based management.

\section{Chronic critical illness}

Chronic critical illness (CCI) represents a devastating challenge in modern surgical critical care where successful interventions have fostered a growing cohort of patients with prolonged dependence on mechanical ventilation and other organ supportive therapies who would previously have succumbed much earlier in the acute phase of critical illness $[8,9]$. An important subset of CCI patients are those who have survived an emergency abdominal operation, but who subsequently require prolonged open abdomen management complicated by persistent peritoneal space infection or colonization, fistula formation, and gastrointestinal (GI) tract dysfunction. In contradistinction to acute GI tract perforation that leads to secondary peritonitis, this latter population instead demonstrates tertiary peritonitis (TP). While secondary peritonitis is optimally managed using operative or percutaneous techniques to achieve source control, TP typically presents few if any opportunities for mechanical intervention. Instead, TP is ideally considered as a failure of host defense rather than a condition best resolved by operative intervention.

\section{Tertiary peritonitis}

Tertiary peritonitis (TP) represents persistent inflammation and colonization as well as intermittent infection in an immune compromised host that develops after what was believed to be a successful attempt at primary source control $[5,15,16]$. This subset of the larger CCI patient population is characterized by non-resolving organ failure that involves multiple systems, but perhaps most importantly, host humoral and cell-mediated defense (Fig. 1) [17]. While sensitive bacteria and fungi are most commonly initially identified in those with secondary peritonitis, microbiome alterations toward nosocomial pathogens are typical for 
Fig. 1 Tertiary peritonitis is severe recurrent or persistent intra-abdominal inflammation after escape from or primary failure to achieve source control in a compromised host. This subset of chronic critical illness is characterized by prolonged systemic inflammation associated with acute respiratory distress syndrome (ARDS) ventilatory failure, renal failure, hypercatabolism, and severe protein-calorie malnutrition

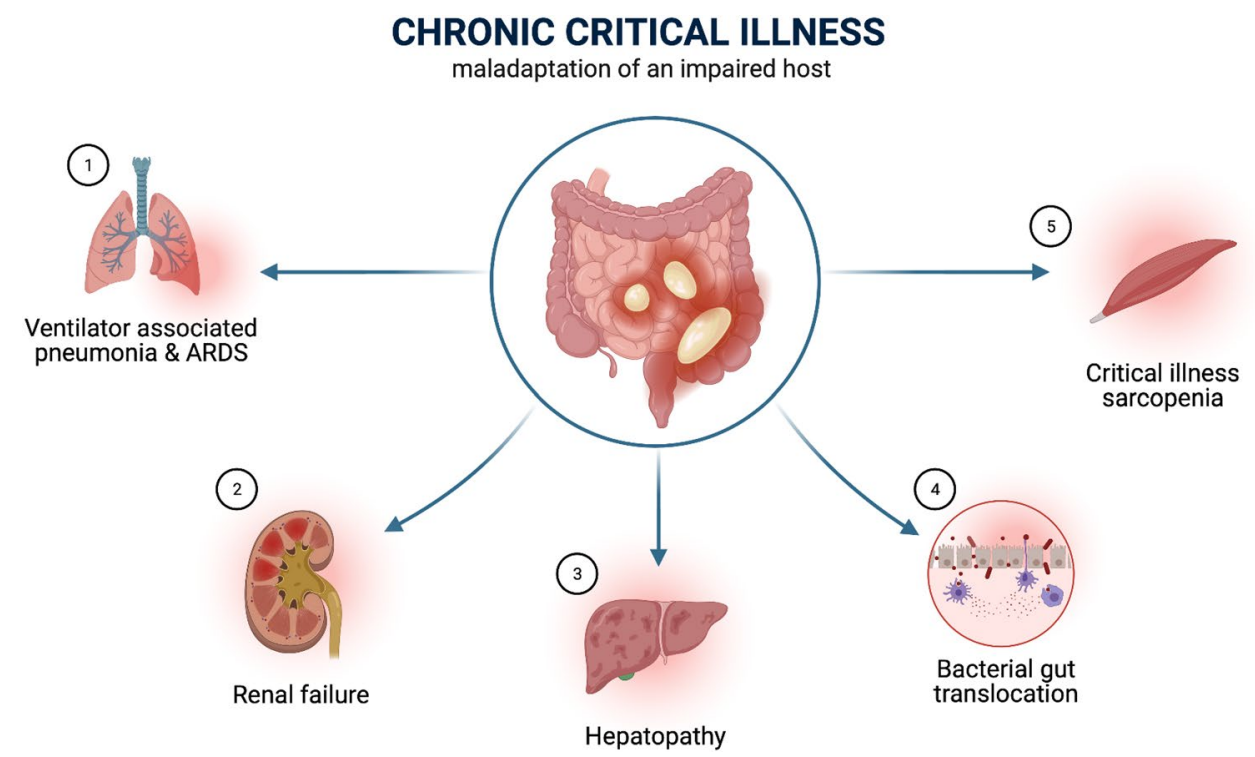

those with TP [18-22]. Repeated courses of therapeutic antibiotics, even when culture directed, drive microbial ecology toward multidrug-resistance organism (MDRO) predominance $[1,6,16,22]$. Defective host defense, exemplified by T-cell exhaustion, disables clearance of MDRO pathogens even when appropriate antibiotic therapy is utilized [23]. Importantly, the clinician must be able to distinguish pathogen invasion from peritoneal space colonization. One useful rubric is that bacteria identified in the open abdomen in the absence of purulence or remote spread (i.e., bacteremia) represent only colonization. Colonization does not require antibiotic management, while purulent peritonitis or remote infection benefits from empiric and then culturedirected therapeutic agent management. The open abdomen that typifies those with TP affords ample opportunity for effluent sampling whether managed using local wound care measures or a negative pressure wound management system.

Open abdomen management using temporary closure devices that employ negative pressure control effluent flow but demonstrates other important physiologic effects that are relevant to the surgeon and intensivist [24]. First, device effluent has been demonstrated to be cytokine rich and may serve as one means of mitigating the overall inflammatory milieu. Second, non-adherent bacteria may also be directed into the effluent as a result of the applied vacuum, decreasing the overall peritoneal space bioburden [25, 26]. It is important to note that dense purulent debris is not well managed using a vacuum-assisted technique that often awaits $48 \mathrm{~h}$ between exchanges, but is better addressed using planned daily lavage and debridement as necessary. Third, especially in those with high volume effluent flow, negative pressure across the open abdominal cavity provides a limited peritoneal dialysis effect, reducing markers of renal function such as serum creatinine. Fourth, this dialysis-like effect may also impact the concentration of therapeutic agents with a high partition coefficient into the peritoneal space. Despite the benefits of open abdomen management with or without negative pressure techniques, there are a variety of well characterized complications for which the clinician must be prepared.

These complications may be conveniently grouped into those that are mechanical, those that impact fluid and electrolyte balance, and those that influence anesthetic, pharmacologic and nutritional support approaches. Mechanical complications include but are not limited to the inability to achieve primary fascial closure leading to a large abdominal wall defect, enterocutaneous (ECF) or enteroatmospheric (EAF) fistula formation, and difficult thermoregulation with an open body cavity. The latter is much reduced using negative pressure wound therapy compared to moist dressings, and generally avoids the use of a active external warming device to support normothermia. Both ECF and EAF are often challenging with regard to fluid loss as well as electrolyte derangement based on external losses as well as the common practice of avoiding luminal nutritional supplementation in favor of intravenous nutritional support (Fig. 2) [27, 28]. Despite ideal management, nonresolving organ failure in TP patients results in mortality in 30-64\% [16, 19, 29]. Therefore, elaborating an integrated approach to the impactable aspects of the complex managment of TP patients helps support recovery despite high acuity or chronic critical illness. This remainder of this review describs such elements and locates them in a multidisciplinary management framework specifically addressing key aspects of surgical management, periprocedural anesthetic considerations, critical care pharmacology, and critical care nutrition support. Since TP patients characterisically flow from those who require secondary peritonitis management, 


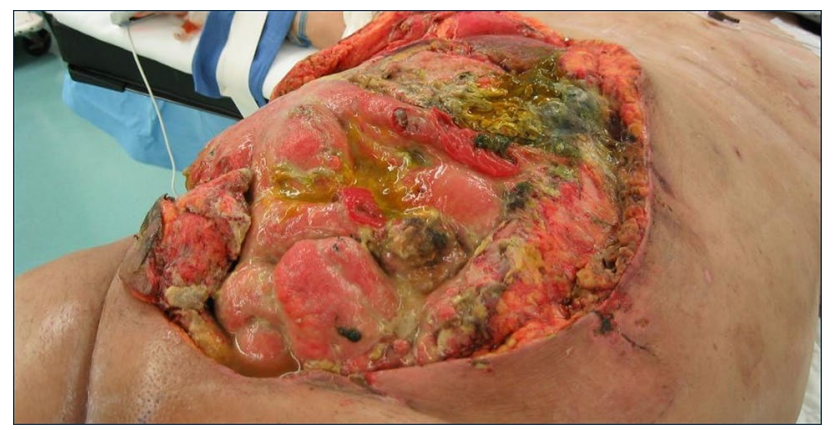

Fig. 2 Frozen open abdomen in tertiary peritonitis, with multiple enteroatmospheric fistulae, demonstrating a patient without options for definitive source control. Clinical image courtesy of Dr. Kaplan. Patient permission obtained for photograph

the surgical elements involved in source control provide a point of embarkation to understand evolution of this disease process as a failure of host defense.

\section{Source control in peritonitis}

Hollow viscus perforation delivers gut microbiome commensals into the peritoneum or retroperitoneum and triggers a local response that trafficks neutrophils, macrophages, and other elements of cell-mediated immunity such as T-cells to the site of injury as part of an adaptive host defense response. Local and remote increases in inflammatory mediators including but not limited to cytokines such as IL-8 and IL-1, pre-formed antibodies, complement, and activated elements of the coagulation cascade are only a small but important part of the competent host response to native or non-native pathogen invasion [30-34]. Related host defense responses include omental adhesion, fibrin deposition, and local inflammation to control pathogen as well as activated enteric enzyme spread. Of course, this approach is more successful when there is hollow viscus wall inflammation that precedes perforation-such as with diverticulitis-where a contained abscess may then develop. The ability of such mechansims to shield healthy tissues is quite limited when perforation occurs rapidly such as with embolic mesenteric ischemia or penetrating injury $[33,34]$. When competent host defense measures are successful in establishing an abscess, bacterial bioburden reduction may require operative therapy if it cannot be achieved using percutaneous drainage. On occasion, some abscesses are quite small, or inaccessible without incurring significant patient morbidity, and may be treated with antibiotic therapy to reduce bacterial bioburden as some antiinfective agents demonstrate abscess penetration [35, 36]. Such an approach is far less robustly supported compared to achieving definitive source control, even if doing so temporarily interrupts intestinal continuity in order to control soilage and allow time for restoration of effective circulatory volume as well as hemostatic and metabolic resuscitation.

\section{Adjuncts to source control}

While abscess cavities benefit from intra-operatively placed or percutaneously placed drains, prophylactic peritoneal drains remain devoid of supporting evidence [37-40]. Pedicled omentum may buttress anastomoses or sites of visceral repair as a vascularized and active host defense barrier [41]. When instestinal or anastomotic viability is uncertain, an open abdomen approach with or without negative pressure wound therapy (NPWT) provides ready access and has been described with and without concomitant continuous peritoneal lavage — an element embraced by devoted adherents but also without clear evidence of benefit [42-45]. When there is densely adherent mucopurulent debris there remains controversy regarding the value of serial re-exploration and peritoneal or organ surface debridement to reduce bacterial bioburden. Animal models of uncontrolled peritonitis have suggested that a strategy of serial planned laparostomies with NPWT may reduce serum IL-6 titers and improved survival compared to primary fascial closure with indwelling intra-peritoneal drainage and on-demand relaparotomy $[25,26]$; an international prospective open-label blockrandomized control trial is currently underway to test these approaches in the critically ill [46].

When visceral edema, packing for hemorrhage control, or disrupted intestinal continuity is encountered during a source control procedure, an open abdominal approach is essential and helps reduce the incidence of intra-andominal hypertension and the abdominal compartment syndrome (ACS). Nonetheless, some of these patients will be unable to achieve primary fascial closure even with approaches that include limited crystalloid fluids, hypertonic saline infusion, and rapid restoration of intestinal continuity [47]. Such patients develop a visceral block of fused hollow organs that are also adherent to the posterior aspect of the anterior abdominal wall; a variety of approaches have been articulated to achieve temporary or more durable closure [24]. Others who are able to be closed will suffer anastomotic leak, and others will develop an ACS leading to urgent or emergent relaparotomy and conversion to open abdomen management. Events such as those detailed above set the stage for progression to tertiary peritonitis, especially when there is concomitant organ injury or failure. 


\section{Organ failure in tertiary peritonitis}

The influence of inadequately controlled, recurrent or persistent intraperitoneal infection on organ failure has been well described. Sepsis related organ failure impacts all organ systems with approximately $50 \%$ of all acute kidney injury in the critically ill being ascribed as a sequela of infection [48]. Both accelerated morbidity and mortality are attributed to non-resolving organ dysfunction in the setting of sepsis and septic shock [49-54]. However, the specific mechanisms by which organ failure management hinders or enables host defense are less clearly defined and outcomes may be substantially driven by patient phenotype as opposed to specific intervention [11]. Nonetheless, common organ support interventions exert influences on host defense with which the clinician should be conversant to help guide care and patient and family or surrogate discussions.

The majority of therapeutic interventions rely on devices that compromise host integument integrity, and as such, generally challenge host defense. Percutaneously placed catheters as well as those that cross otherwise sterile divides-such as the larynx - typify access devices that increase infection risk. Indeed, invasive mechanical ventilation engenders ventilator associated event and well as ventilator-associated pneumonia risks, both of which may prolong ICU length of stay, and increase mortality [55-58]. In particular, those with the Acute Respiratory Distress Syndrome (ARDS), or those at risk of ARDS, may have some of their outcome related to their inflammatory profile with worse outcomes linked to a hyperinflammatory state [10]. Those with ARDS often also demonstrate AKI, many of whom require renal support. Patients with septic shock who undergo continuous renal support may demonstrate a rapid improvement in both hemodynamics and outcomeobservations believed to be in part related to inflammatory mediator clearance in device effluent that have been colloquially termed "blood purification in sepsis" [6]. Specifically, improvements in antigen-presenting capability, leukocyte trafficking and responsiveness, as well as oxidative burst and phagocytosis, may be identified in some septic patients while receiving continuous renal support $[59,60]$. Patient selection for this intervention remains unclear but may be ultimately informed by subphenotype identification as noted above for those with ARDS. Both invasive mechanical ventilation and continuous renal support techniques are common in those with septic shock as well as those with tertiary peritonitis. As such, they represents bedside procedures that often involve sedation and analgesia that is titrated to patient-centered goals by the ICU team. However, some such patients require episodic care in the Operating Room requiring that the entire team anticipates their unique needs.
Specific anesthesia considerations in tertiary peritonitis

Tertiary peritonitis presents unique challenges to the anesthesiologist for a host of reasons that impact OR management. These include but are not limited to: complex pre-operative evaluation, challenges in obtaining informed consent, determining the optimal location for exploration with the surgical team, as well as the intra-operative management of a patient with systemic inflammation, sepsis, or non-resolving multiple organ dysfunction. Post-operative concerns include anticipating postoperative care needs-including those during intra-facility transport-that may encompass invasive monitoring, hemodynamic support, as well as mechanical ventilation (not hand ventilation). Accordingly, the anesthetic care for patients with tertiary peritonitis may be conveniently grouped into three major phases (Fig. 3).

\section{Pre-operative evaluation and decision-making}

When tertiary peritonitis is complicated by a surgical emergency, there is often limited time for the anesthesia team to conduct a thorough pre-operative assessment. A focused history and physical is warranted, with attention to the patient's cardiopulmonary stability (e.g., heart rate and rhythm, blood pressure and vasoactive infusions, acute respiratory distress or failure), key laboratory studies (e.g., $\mathrm{pH}, \mathrm{pCO}_{2}$, lactate, serum potassium, creatinine, hemoglobin), airway access history, and vascular access. These data will facilitate developing an anesthetic plan that preserves cardiopulmonary stability to the greatest extent possible, and will aid the anesthesia team to rapidly address unanticipated deterioration in the OR. The clinical data will also help the anesthesiologist, in partnership with the surgeon, decide whether the patient's case should be conducted in the operating room or at the bedside in the ICU. Finally, when possible and in concordance with institutional policies, informed consent for anesthesia care should be obtained. Often, the patient with tertiary peritonitis cannot provide informed consent due to critical illness or therapeutic agent infusion. Therefore a consent discussion with the patient's health care proxy decision-maker is essential. Such a conversation should stress the high risk but necessary nature of the surgical procedure as well as a discussion of the potential risks of anesthetic care.

\section{Intra-operative management}

Vascular access is critical to the intra-operative management of the patient with tertiary peritonitis. Ideally, this access is in place prior to surgery, but additional access may need to be obtained. Central line placement may be necessary if 
Fig. 3 Triphasic approach to anesthesia considerations in tertiary peritonitis. 1 Focused evalution of cardiopulmonary stability, airway access history, and vascular access. 2 Planned plasma volume expansion, or pre-emptive administration of vasopressor agents, agile and vigilant cardiovascular monitoring and intervention. 3 Structured hand-offs so that all team members are present at the same time has been evaluated and serves as a best practice to ensure information fidelity. (HATRICC Trial) [65, 66]

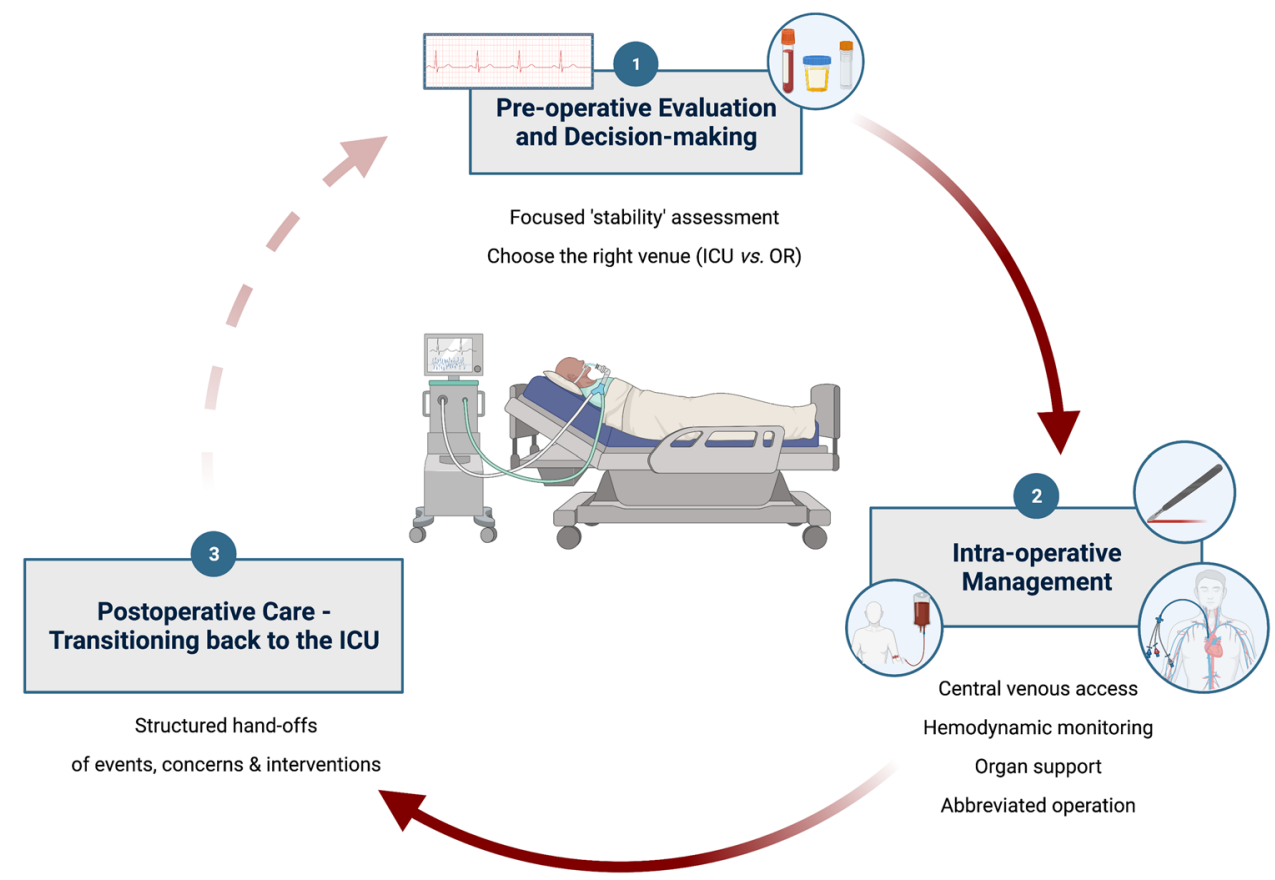

vasopressor use is expected (depending on dose and institutional policy), but sterile line placement could present an unacceptable delay to the start of surgery for the patient with septic shock. Often, anesthesiologists "make do" with the vascular access present on arrival to the operating room. Indeed, there is a growing body of evidence that low to middose vasopressors may be safely delivered via secure peripheral access. Similarly, arterial line blood pressure monitoring offers moment-to-moment titration data, but there may be insufficient time to place such a line prior to the start of surgery. Consideration for placement during the operative procedure should be undertaken.

General anesthesia with chemical neuromuscular blockade is likely the best approach to manage the patient with tertiary peritonitis. If the patient is endotracheally intubated prior to surgery, anesthesia can be induced with inhaled anesthetics. There are specific considerations that should influence induction agent selection, particularly in the setting of concomitant established or evolving organ failure. There is some evidence that agents such as etomidate, a potent suppressant of adrenal steroidogenesis, effectively induces reversible pharmacological adrenalectomy, that may lead to hemodynamic collapse and further complicate complex management [61-63]. Additionally, based upon immobility and potential muscle breakdown as well as the common presence of some degree of AKI, depolarizing neuromuscular blocking agents such as succinylcholine that may induce hyperkalemia are best avoided in favor of non-depolarizing agents. Finally, neuromuscular blocking agents that require hepatic metabolism should be supplanted by $c i s$-atracurium that instead undergoes plasma metabolism. Hepatically cleared agents may demonstrate prolonged neuromuscular blockade due to delayed clearance.

If the patient requires intubation before surgery, the anesthesia team should be prepared for the patient to experience an acute decrease in cardiac output, and therefore blood pressure, upon the induction of general anesthesia. This fall in cardiac output can be attributed to diminished preload due to induction agent associated vasodilation and may be exacerbated by sepsis-driven precapillary arteriolar sphincter relaxation. Planned plasma volume expansion, or pre-emptively administering vasopressor agents (e.g., phenylephrine, ephedrine, epinephrine) are successful strategies to avoid induction agent associated hypotension, which in severe cases, can lead to cardiac arrest. Once general anesthesia is induced, vigilance is needed to monitor for changes in cardiac output related to vasodilation, fluid losses, and surgical manipulation. This monitoring is similar to that for any laparotomy, but patients with tertiary peritonitis have less physiologic reserve due to hypercatabolism, inflammation, and concomitant non-resolving organ dysfunction leading to an accelerated risk for cardiovascular instability. As with all complex intra-operative care, frequent communication between the anesthesia and surgical teams is essential to ensure as ideal a patient outcome as possible.

If the patient's surgery occurs in the intensive care unit, monitoring and providing sedative agents will usually be directed by the intensive care unit team. It is important to designate at least one staff member (often a critical care nurse) to monitor and document the patient's vital signs frequently (e.g., every 2-5 min). Continuous 2-lead electrocardiogram monitoring and pulse oximetry are required 
monitors for bedside laparotomy, as is continuous blood pressure measurement with an arterial line. Coordination with OR scrub or circulator staff may be required if the ICU team cannot serve in these roles. Sites where bedside reexploration is common often train staff to serve in those capacities. The decision for bedside re-exploration may be driven by the concomitant need for advanced ventilation techniques that augment transport risks [64].

\section{Postoperative care-transitioning back to the ICU}

Most patients with tertiary peritonitis will benefit from ICU management after surgery given their needs for hemodynamic monitoring or support, as well as concomitant organ failure management. It is important that the anesthesia care team communicate with the post-operative care team about intra-operative events including but not limited to the status of new or pre-existing devices, airway or pulmonary concerns, and interventions to address cardiovascular performance or stability. Such hand-offs between care teams are critical for active management, especially in complex patients. Structuring hand-offs so that all team members are present at the same time has been evaluated and serves as a best practice to ensure information fidelity [65, 66].

One of the more challenging aspects of care is the management of therapeutic medications in terms of appropriate therapeutic agent dosing and surveillance for untoward medication interactions.

\section{Clinical pharmacy considerations}

Appropriate antimicrobials should be administered without delay in patients with suspected tertiary peritonitis [7, 67, 68]. The selection of optimal antimicrobial regimens will consider the infective organism, pharmacokinetic changes in the critically ill, and potential adverse effects of the antimicrobial as well as data derived from the local antibiogram. Furthermore, antimicrobial stewardship is imperative to mitigate the potential sequalae of prolonged broad-spectrum regimens, namely the development of multidrug-resistant organisms (MDRO) as well as superinfection with organisms such as Clostridium difficile or fungi.

Physiologic aberrations occur in the critically ill and injured patient throughout their illness resulting in pharmacokinetic and pharmacodynamic variability. Underlying chronic illnesses as well as acute or non-resolving organ dysfunction managed using extracorporeal support techniques will influence the relationship between therapeutic agent dose and the intended response. Therefore, empiric dose selection as well as subsequent dose management may be challenging [69-71]. Furthermore, dosing recommendations in critically ill patients are frequently extrapolated from studies in healthy volunteers and other non-critically ill patient populations. As such, these extrapolations may lead to unintended toxicities or therapeutic failures resulting from alterations in bioavailability, volume of distribution, and clearance (Fig. 4).

Many antimicrobials used to treat tertiary peritonitis are hydrophilic molecules (e.g., beta-lactams, aminoglycosides, vancomycin) making them susceptible to significant pharmacokinetic alterations during the course of critical illness [69-71]. For example, large volume resuscitation, capillary leak syndrome, enhanced hepatic metabolism, augmented renal clearance, and extracorporeal support may all lead to suboptimal serum concentrations when standard dosing regimens are employed. Importantly, diminished hepatic blood flow, decreased protein concentration, and acute kidney injury will each increase the risk of toxicities unless standard doses are adjusted. Additionally, since gastrointestinal absorption can be appreciably compromised in the critically ill, the intravenous route of drug administration is often preferred to ensure rapid onset of action and complete antimicrobial bioavailability. Given these and other uncertainties that may impact the therapeutic effect of antimicrobials, therapeutic drug monitoring can be used to ensure adequate therapeutic targets while monitoring for potential toxicities $[72,73]$. Therapeutic drug monitoring is only widely available for aminoglycosides and vancomycin, and is only selectively available for beta-lactam antimicrobials.

Pathogens associated with tertiary peritonitis include gram-negative aerobes (e.g., Pseudomonas, Enterobacter, Acinetobacter), enteric anaerobes, gram-positive bacteria (e.g., coagulase-negative Staphylococcus species, methicillin-resistant I, and I), and Candida species (Fig. 5) [7, 68, 74]. When acute infection is suspected, empiric therapy must therefore be broad with subsequent tailoring based upon culture and sensitivity profiling. However, the open peritoneal space cannot be sterilized and colonization is expected, often including MDRO. Bacterial antimicrobial resistance is common and of particular concern in patients that have been acutely hospitalized for greater than $48 \mathrm{~h}$ (especially in an ICU), recently completed a course of therapeutic antibiotics, or have completed an inpatient acute care stay or received home wound care or dialysis within the preceding 90 days [68]. Gram-negative bacilli that demonstrate inducible or constitutive extended spectrum beta-lactamase activity, or carbapenemase activity (i.e., carbapenem-resistant Enterobacteriaceae) present management challenges in patients with tertiary peritonitis, especially when there is infection remote from the peritoneal space such as bacteremia, catheter-related infection, cystitis, or pneumonia [75, 76].

The optimal duration of antimicrobial therapy for tertiary peritonitis in the critically ill remains elusive. Tertiary peritonitis is different from secondary peritonitis in that the former represents a failure of host defense while the 
Fig. 4 Pharmacologic dosing recommendations in critically ill patients, extrapolated from studies in healthy volunteers and other non-critically ill patient populations, may lead to unintended toxicities or therapeutic failures resulting from alterations in bioavailability, volume of distribution, and clearance. Diminished enterohepatic blood flow, decreased protein concentration, and acute kidney injury will each increase the risk of toxicities unless standard doses are adjusted. Additionally, since gastrointestinal absorption can be appreciably compromised in the critically ill, the intravenous route of drug administration is often preferred to ensure rapid onset of action and complete antimicrobial bioavailability

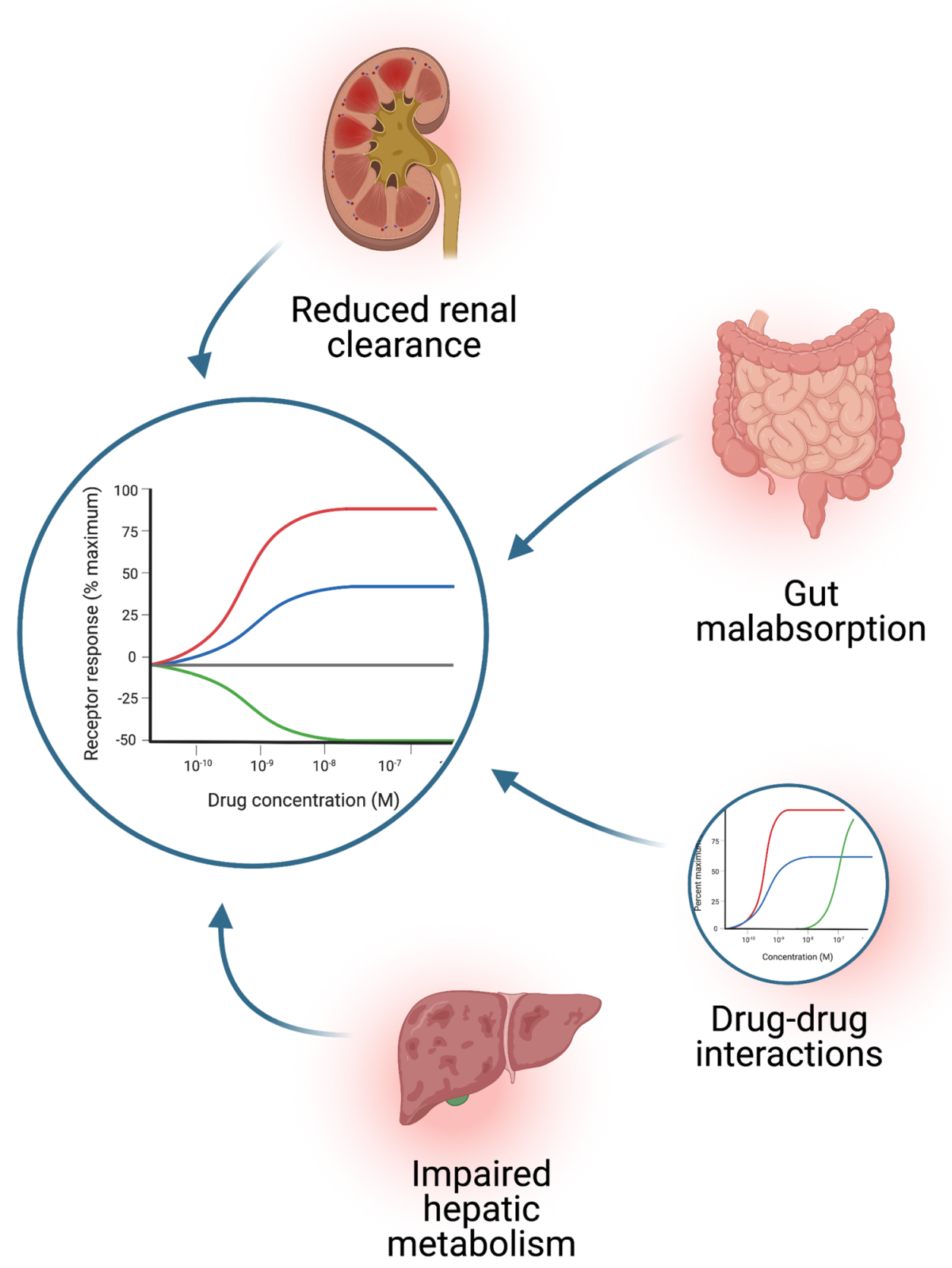

latter provides an opportunity to pursue source control. For patients with adequate source control the Surgical Infection Society and the Infectious Diseases Society of America recommend limiting antimicrobial therapy to 4-7 days [7, 68]. Short course (4 days) antimicrobial therapy is appropriate in non-critically ill patients with intraabdominal infections and adequate source control [77]. A more prolonged, 7-days course may be reserved for critically ill patients, as well as those in whom source control is problematic [76]. Interestingly patients with bacteremia or the need for percutaneous drainage had higher failure rates when randomized to an 8 -days treatment course compared to 15 days, suggesting that some patient characteristics-principally difficult source control—may warrant an extended course of therapy [76].

Procalcitonin may be useful in some settings to help reduce the duration of antimicrobial exposure and an algorithm for its use has been articulated [78]. However, its use in tertiary peritonitis is limited by falsely low concentrations in the setting of fungal infection, abscess formation, or recurrent infection [79]. Most importantly, no antimicrobial therapy is required for colonization of the open abdomen in patients with tertiary peritonitis; directed therapy is, on the other hand, essential for remote infection and should be limited to those specific conditions. Those with tertiary 


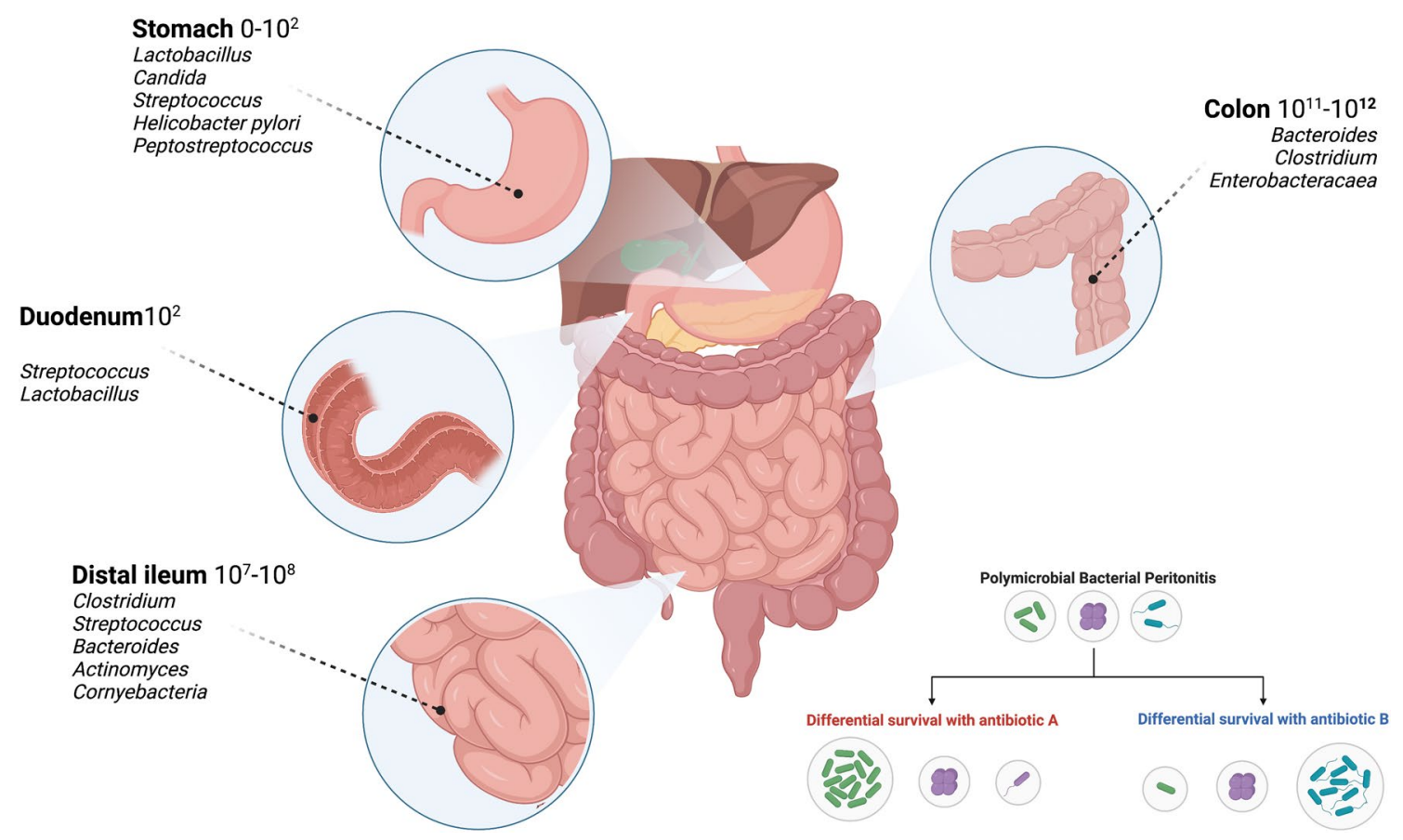

Fig. 5 Pathogens associated with tertiary peritonitis include Gramnegative aerobes (e.g., Pseudomonas, Enterobacter, Acinetobacter), enteric anaerobes, Gram-positive bacteria (e.g., coagulase-negative species, methicillin-resistant Staphylococcus aureus, and Enterococcus), and Candida species. Bacterial antimicrobial resistance is

peritonitis generally demonstrate a hypercatabolism driven inflammatory profile marked by a high C-reactive protein concentration that may render using standard markers of nutritional adequacy difficult to apply. Therefore, the team should elaborate a comprehensive nutritional plan to address the unique elements of the patient with tertiary peritonitis [80].

It is important to recognize variations in team membership that occur in different world regions. While some countries, such as the US, is often well resourced in terms of Clinical Pharmacists (PharmD's) who round with and are integrated into the critical care team, this may not be the case in other geographies. In the absence of an integrated team clinical pharmacist, dialog with the hospital Pharmacy is prudent for complex care. For instance, in high dependency units in many countries, the Clinical Pharmacist evaluates orders and interrogates the medication administration profile for untoward interactions and opportunities for pharmacokinetic or pharmacodynamic assessment. Their findings may be communicated to the team asynchronous with bedside rounds representing an opportunity for specific queries to be made of the Pharmacy staff if unanticipated care demands rapidly evolve. Regardless of the locations where medication safety and interaction are assessed, Pharmacists serve a dynamics role in securing safe and effective care. common and of particular concern in patients that have been acutely hospitalized for greater than $48 \mathrm{~h}$ (especially in an ICU), recently completed a course of therapeutic antibiotics, or completed an inpatient acute care stay or received home wound care or dialysis within the preceding 90 days

\section{Nutrition for recovery}

Nutrition therapy is an important aspect of care in the patient with tertiary peritonitis (TP). TP patients may frequently be classified as having chronic critical illness (CCI) due to late or recurrent intra-abdominal infection, prolonged systemic inflammation, and non-resolving organ dysfunction. CCI, identified as a distinct syndrome in 2012, is defined by ICU length of stay greater than 14 days, recurrent infection, and persistent organ dysfunction [81]. It is apparent that the critical care team must recognize and respond to changes in the catabolic state of the patient that are both time as well as diagnosis related throughout the timeline of a patient's ICU care.

Nutrition evidence-base ICU guidelines [80, 82] recommend early nutrition progressively implemented within $48 \mathrm{~h}$ of admission, and advancing to goal as the patient reaches the end of the early phase of critical illness (Fig. 6). While this approach is appropriate for the critically ill with a projected rapidly resolving course, it is less ideal for those with TP. In those with CCI, a group that includes patients with $\mathrm{TP}$, malnutrition with accelerated lean body mass loss is not abrogated using a standard approach [83]. Despite a paucity of evidence, the current recommendation for CCI patients support luminal nutritional using a high-protein formulation 
Fig. 6 Failure or inability to utilize enteral nutrition, in combination with inflammation and interstitial edema, results in bacterial translocation. Prolonged fasting also depletes the luminal secretions containing brush-border enzymes that are necessary for efficient absorption of nutrients

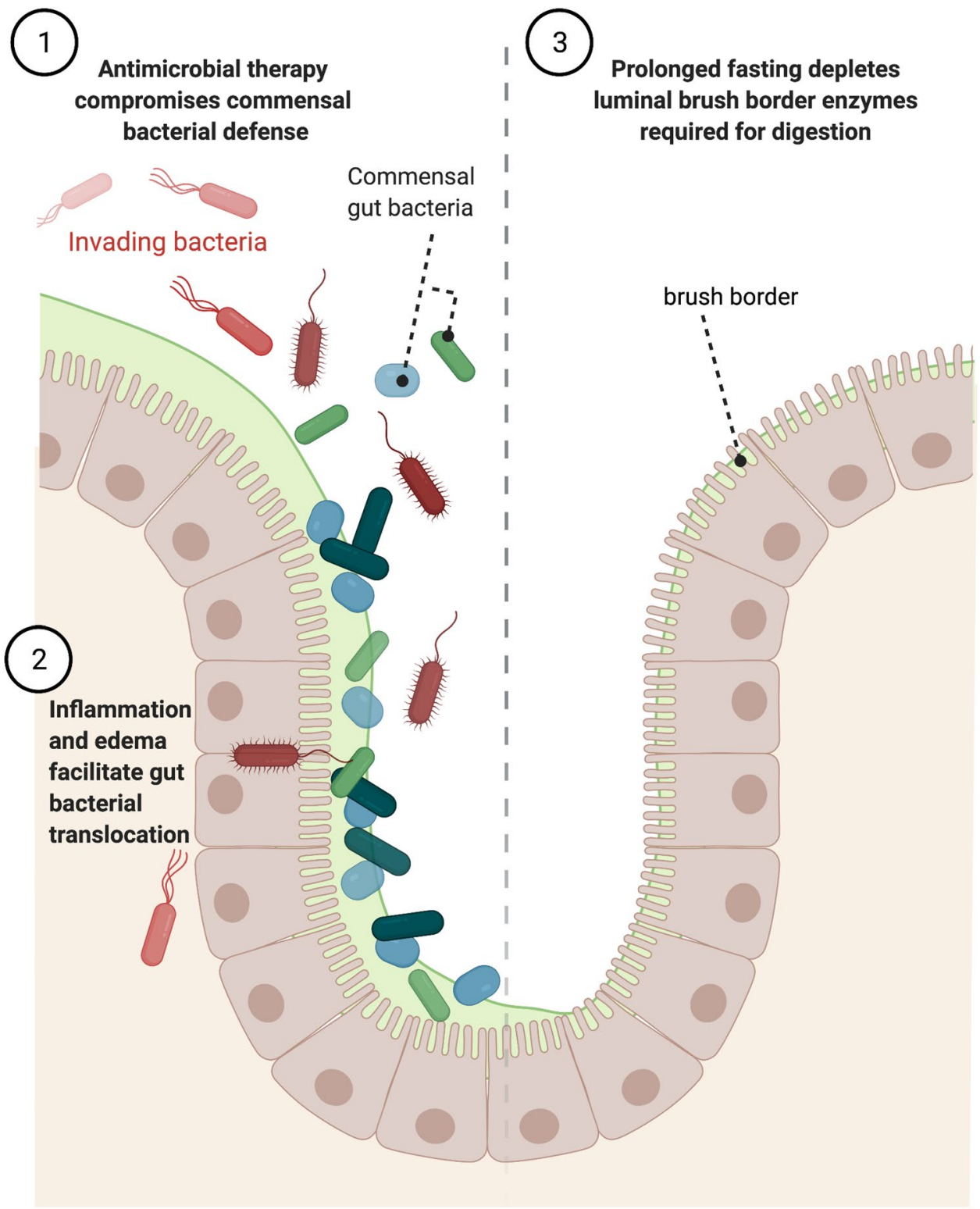

and, if feasible, a progressive resistance-based exercise program [80]. Protein administration at greater than $2 \mathrm{~g} / \mathrm{kg} \mathrm{bw}$ per day is typical for this patient population to address both hypercatabolism and external protein loss across the open peritoneal space.

Several studies have attempted to define an "average" protein loss across the open abdomen to inform the nutritional prescription. Such studies have been hampered by heterogeneity in patient type, disease process, plasma volume, and timing. Estimated include $3.5 \mathrm{~g} \mathrm{~N}_{2} / 24 \mathrm{~h}$ in those with emergency general surgery or injury [84] up to $2.9 \mathrm{~g}$ protein/dl of effluent [85]. More recent estimates suggest $1.5 \mathrm{~g}$ protein/dl of effluent [80]. Regardless of the estimate used, it is essential to recall that all measures of protein catabolism (i.e., UUN, indirect calorimetry) or nutritional adequacy (i.e., prealbumin controlled for C-reactive protein) will not account for accelerated external losses across the open peritoneal space.

A structured nutritional support approach may be informed by data obtained in related populations such as those with thermal injury or malignancy-related cachexia as persistent inflammation, lean body mass catabolism, and anabolic resistance are characteristic. Those specific settings have delineated hormonal changes during critical illness and may guide adjunctive therapies that address elevated endogenous catecholamine concentration, as well as hormonal milieu modulation. Adjunctive therapy with specific supplements may work synergistically with standard nutritional prescriptions and are ideally targeted to specific physiologic abnormalities and to achieve trackable outcomes. 
To mitigate lean body mass catabolism after thermal injury, burn care programs treat patient with accelerated protein intake combined with an early exercise program. In the chronic phase of burn recovery, however, anabolic interventions, including oxandrolone and propranolol, appear helpful [86-88]. This combination leverages what is known about hormonal changes over the course of critical illness, especially those related to anterior pituitary. Acutely, growth hormone $(\mathrm{GH})$, thyrotrophin (TSH), and corticotrophin (ACTH) secretory activity is amplified, whereas active thyroid hormone and insulin-like growth factor-1 (IGF-1) levels are suppressed. Therefore, early use of anabolic agonists during burn patient care such as oxandrolone are likely to be ineffective during a period of elevated GH concentration. This findings may be patient population specific, as those with CCI demonstrate suppressed pulsatile secretion of GH, $\mathrm{TSH}$, and ACTH which results in low circulating levels of IGF-1, IGF binding protein-3, and thyroid hormones [89]. Suppressed pulsatile hormone secretions may be partially responsible for the chronic fatigue and muscle weakness of CCI [90]. Since CCI is commonly linked with sepsis, extrapolation from septic patient intervention data seems reasonable. Only quite old data that suggests increased mortality with GH supplementation as a means to overcome suppressed pulsatility is available [91]. Therefore, there is no current recommendation to use hormonal agonists in those with CCI, including patients with TP.

The catabolic state associated with malignancy-driven cachexia is similar to that in sepsis associated CCI and is commonly linked with a persistent expansion of myeloidderived suppressor cells that secrete arginase-1 [92]. Arginine deficiency-induced T-lymphocyte dysfunction impairs cell-mediated immunity due to T-cell functional suppression and is associated with an increased risk of infection. In addition, arginine is synergistic with the branch-chained amino acid (BCAA) leucine, in that they both stimulate anabolism via the mammalian target of rapamycin (mTOR) signaling pathway. Unfortunately, mTOR is downregulated during acute sepsis or septic shock. However, it is possible that supplemental arginine, leucine, and potentially $\beta$-hydroxy $\beta$-methylbutryate (HMB, a leucine metabolite) may support anabolism, lean body mass, strength and endurance albeit at a reduced rate compared to health [93]. Milieu modification has been demonstrated with other supplements including omega-3-fatty acids (FA).

Omega-3 FAs augment adaptive immune responses and decrease inflammatory biomarkers in cancer patients [94]. Specialized pro-resolving mediators (SPMs) derived from omega-3 FAs offer a mechanism that underpins their benefit in a variety of disease states. SPMs play a key role in dampening the inflammatory response without inducing concomitant immunosuppression. Moreover, they promote tissue regeneration and potentially limit organ injury by impeding inflammation-associated fibrosis [95, 96]. Such effects could reasonably help improve CCI patient trajectories, by supporting recovery while reducing systemic inflammation.

Other supplements such as vitamin D seem devoid of similar benefit in the critically ill, and are therefore not routinely recommended for those with CCI or TP to improve mortality [97]. However, when vitamin D was given in conjunction with a high-protein, BCAA-containing oral supplement to deficient older adults, lean body mass was maintained with improved walking performance [98, 99]. In combination, providing supplemental enteral arginine, omega-3 FAs, HMB, and vitamin D as part of a multifaceted nutritional prescription seems reasonable and safe in the critically ill to improve performance indicators other than mortality [80, 82]. To a similar end, routine supplementation with vitamin $\mathrm{C}$ is essential to support collagen cross-linking as new tissue is elaborated-especially since the intake of citrus fruit is rather limited in those being nourished using luminal nutritional support. Vitamin $\mathrm{C}$ concentrations vary by specific formula and may provide much less than $1 \mathrm{~g}$ over a $24 \mathrm{~h}$ course even when the nutritional prescription achieves the protein and non-protein or total calorie goals for a given patient.

Since the majority of patients with TP have had limited oral intake, these patients should be assumed to have absent luminal briush border enzyme systems to process complex nutrients. Therefore, initial luminal nutritional supplementation should leverage the direct absorption capabilities of an elemental formulation while the intenstinal luminal glycocalyx is reestablished. Otherewise, diarrhea from malabsorption is a common consequence that may also derange fluid and electrolyte homeostasis in a preventable fashion. Similar to clinical pharmacy integration, not all centers have integrated registered dietitians as part of the rounding team, but routine consultation for patients starting on new luminal or intravenous nutritional support offers opportunities for nutrition prescription refinement, and impact assessment on lean body mass, nitrogen balance, and organ function.

\section{An integrated multidisciplinary approach to care}

Finally, no comprehensive plan for care of the patient with tertiary peritonitis would be complete without evaluating subsequent care. In the improving patient, early physical therapy and rehabilitation are essential to mitigate ICU weakness, loss of skeletal muscle mass, deconditioning, as well as the cognitive impariement that characterizes the post-intensive care syndrome (PICS). Post-ICU clinics are increasingly common to address this vulnerable patient population, identify those in need of focused rehabilitation, and to enroll them in peer support groups [100]. These elements 
are critically important especially in those transferred to a tertiary or quaternary center and then repatriated to their home community. Uncompensated caregivers are often left addressing ongoing care and may not be underpinned by adequate resources [101]. While we have identified sequelae of critical illness in patients and family members, we are now intensively exploring the impact of prolonged critical care in ICU professionals as well. Burnout syndrome has been brought to the fore by COVID-19 in the wake of a 2016 call to action that highlighted this syndrome in critical care clinicians $[102,103]$. While we diligently work to rescue the critically ill, we must also ensure that we do not fail to rescue those invested in caring for such patients regardless of their duration in the ICU [104].

While not all patients will survive their complex ICU or hospital stay, mortality prognostication may be challenging to aid family or surrogate decision-making. On the other hand, predicting care interventions that may be required to pursue a survival course are more straightforward. Sharing such information with the patient (if they are able) and the family or surrogate defines a change in life circumstance that may be difficult to place into perspective or digest. In these circumstances, palliative care medicine (PCM) consultation is invaluable. Additionally, having a clinician who is not involved in daily care decision-making may provide comfort for faily members or surrogates, especially in those whose care does not follow a standard pathway. PCM consultation does not diminish the importance of providindg primary palliation that is the purview of every intensivist, but instead provides continuity for symptom management and goals of care discussions that are both complex and time consuming. For those with an untoward trajectory, having an ally in prioritizing life quality over life quantity is particularly important. Patients with CCI or TP represent patients whose ICU length of stay is long, challenging, and fraught with a variety of challenges. However, having an internally consistent integrated approach to the problematic elements of care helps ensure critical care success, or recognize when care is unlikely to achieve the patients goals and a transition toward alternate care goals is most appropriate.

\section{Conclusions}

Tertiary peritonitis represents a unique challenge to the surgeon, intensivist, and ICU multiprofessional team. This bioaltered host, often in multiorgan failure, represents a subset of patient where failed source-control transitions into chronic critical illness. Targeted nutritional and pharmacologic interventions, antimicrobial stewardship, a thoughtful anesthesia and sedation strategy, and goal-directed repair of organ failures will support patient recovery. Early, planned palliative care medicine consultation is a key element in supporting patient and family-centered care while identifying and delivering goal concordant care.

Author contributions GAB and LK conceived the idea, drafted the manuscript, and participated in its critical review and final approval. ALD, MLF, and BT drafted the manuscript and participated in its critical review and final approval.

Funding No financial support or funding was received for the presented work.

\section{Declarations}

Conflict of interest The authors have no conflicts of interest to disclose.

\section{References}

1. Waele JD, Lipman J, Sakr Y, Marshall JC, Vanhems P, Groba $\mathrm{CB}$, et al. Abdominal infections in the intensive care unit: characteristics, treatment and determinants of outcome. BMC Infect Dis. 2014; $14: 420$

2. Vincent J-L, Rello J, Marshall J, Silva E, Anzueto A, Martin $\mathrm{CD}$, et al. International study of the prevalence and outcomes of infection in intensive care units. JAMA. 2009;302:2323-9.

3. Inui T, Haridas M, Claridge JA, Malangoni MA. Mortality for intra-abdominal infection is associated with intrinsic risk factors rather than the source of infection. Surgery. 2009;146:654-62.

4. Schein M, Marshall J. Source control for surgical infections. World J Surg. 2004;28:638-45.

5. Sawyer RG. Source control, a guide to the management of surgical infections. Berlin: Springer; 2003. p. 341-7.

6. Kaplan LJ, May AK, Napolitano LM. Source control and supporting therapeutics: integrating bacterial invasion, host defense, and clinical interventions with source control procedures. In: Martin ND, Kaplan LJ, editors. Principles of adult surgical critical care. Springer: Berlin; 2016. p. 267-79.

7. Solomkin JS, Mazuski JE, Bradley JS, Rodvold KA, Goldstein EJC, Baron EJ, et al. Diagnosis and management of complicated intra-abdominal infection in adults and children: guidelines by the surgical infection society and the infectious diseases society of America. Clin Infect Dis. 2010;50:133-64.

8. Rhodes A, Evans LE, Alhazzani W, Levy MM, Antonelli M, Ferrer R, et al. Surviving sepsis campaign: international guidelines for management of sepsis and septic shock: 2016. Intensive Care Med. 2017;43:304-77.

9. Heffernan DS, Evans HL, Huston JM, Claridge JA, Blake DP, May AK, et al. Surgical infection society guidance for operative and peri-operative care of adult patients infected by the severe acute respiratory syndrome coronavirus-2 (SARS-CoV-2). Surg Infect. 2020;21:301-8.

10. Kitsios GD, Yang L, Manatakis DV, Nouraie M, Evankovich J, Bain W, et al. Host-response subphenotypes offer prognostic enrichment in patients with or at risk for acute respiratory distress syndrome*. Crit Care Med. 2019;47:1724-34.

11. Seymour CW, Kennedy JN, Wang S, Chang C-CH, Elliott CF, $\mathrm{Xu} \mathrm{Z}$, et al. Derivation, validation, and potential treatment implications of novel clinical phenotypes for sepsis. JAMA. 2019;321:2003-17. 
12. Efron PA, Mohr AM, Bihorac A, Horiguchi H, Hollen MK, Segal MS, et al. Persistent inflammation, immunosuppression, and catabolism and the development of chronic critical illness after surgery. Surgery. 2018;164:178-84.

13. Baethge C, Goldbeck-Wood S, Mertens S. SANRA-a scale for the quality assessment of narrative review articles. Res Integr Peer Rev. 2019;4:5.

14. Higgins JPT, Thomas J, Chandler J, Cumpston M, Li T, Page MJ, Welch VA (editors). Cochrane Handbook for Systematic Reviews of Interventions. 2nd Edition. Chichester (UK): John Wiley \& Sons, 2019.

15. Nathens AB, Rotstein OD, Marshall JC. Tertiary peritonitis: clinical features of a complex nosocomial infection. World J Surg. 1998;22:158-63.

16. Evans HL, Raymond DP, Pelletier SJ, Crabtree TD, Pruett TL, Sawyer RG. Tertiary peritonitis (recurrent diffuse or localized disease) is not an independent predictor of mortality in surgical patients with intraabdominal infection. Surg Infect. 2001;2:255-65.

17. Tellor B, Skrupky LP, Symons W, High E, Micek ST, Mazuski JE. Inadequate source control and inappropriate antibiotics are key determinants of mortality in patients with intra-abdominal sepsis and associated bacteremia. Surg Infect. 2015;16:785-93.

18. Panhofer P, Izay B, Riedl M, Ferenc V, Ploder M, Jakesz R, et al. Age, microbiology and prognostic scores help to differentiate between secondary and tertiary peritonitis. Langenbeck's Arch Surg. 2009;394:265-71.

19. Panhofer P, Riedl M, Izay B, Ferenc V, Ploder M, Jakesz R, et al. Clinical outcome and microbial flora in patients with secondary and tertiary peritonitis. Eur Surg. 2007;39:259-64.

20. Weiss G, Meyer F, Lippert H. Infectiological diagnostic problems in tertiary peritonitis. Langenbeck's Arch Surg. 2006;391:473-82.

21. Pattonieri V, Perrone G, Tarasconi A, Abongwa HK, Catena F. Infections in surgery, prevention and management. Hot Top Acute Care Surg Trauma. 2021. https://doi.org/10.1007/978-3030-62116-2_12.

22. Fomin P, Matviychuk O, Korniychuk O. Microbiological spectre of tertiary peritonitis as a component of its diagnostics and treatment. Eureka Heal Sci. 2017;6:10-8.

23. Guinault D, Nicolau-Travers M-L, Silva S, Cointault O, Daniau B, Bello AD, et al. Expression of exhaustion markers on CD8+ T-cell patterns predict outcomes in septic patients admitted to the ICU. Crit Care Med. 2021. https://doi.org/10.1097/CCM.00000 00000005047 (Publish Ahead of Print).

24. Poillucci G, Podda M, Russo G, Perri SG, Ipri D, Manetti G, et al. Open abdomen closure methods for severe abdominal sepsis: a retrospective cohort study. Eur J Trauma Emerg S. 2020. https://doi.org/10.1007/s00068-020-01379-0.

25. Kirkpatrick AW, Roberts DJ, Faris PD, Ball CG, Kubes P, Tiruta $\mathrm{C}$, et al. Active negative pressure peritoneal therapy after abbreviated laparotomy. Ann Surg. 2015;262:38-46.

26. Xiao Z, Wilson C, Robertson HL, Roberts DJ, Ball CG, Jenne $\mathrm{CN}$, et al. Inflammatory mediators in intra-abdominal sepsis or injury—a scoping review. Crit Care. 2015;19:373.

27. Latifi R, Joseph B, Kulvatunyou N, Wynne JL, O'Keeffe T, Tang A, et al. Enterocutaneous fistulas and a hostile abdomen: reoperative surgical approaches. World J Surg. 2012;36:516-23.

28. D'Hondt M, Devriendt D, Rooy FV, Vansteenkiste F. Systemic peritoneal cavity lavage: a new strategy for treatment of the open septic abdomen. Acta Chir Belg. 2016;107:583-7.

29. Buijk S, Bruining H. Future directions in the management of tertiary peritonitis. Intensive Care Med. 2002;28:1024-9.

30. Novotny AR, Reim D, Assfalg V, Altmayr F, Friess HM, Emmanuel $\mathrm{K}$, et al. Mixed antagonist response and sepsis severity-dependent dysbalance of pro- and anti-inflammatory responses at the onset of postoperative sepsis. Immunobiology. 2012;217:616-21.

31. Sartelli M, Catena F, Saverio SD, Ansaloni L, Malangoni M, Moore EE, et al. Current concept of abdominal sepsis: WSES position paper. World J Emerg Surg. 2014;9:22.

32. Heffernan DS, Monaghan SF, Thakkar RK, Tran ML, Chung C-S, Gregory SH, et al. Inflammatory mechanisms in sepsis. Shock. 2013;40:122-8.

33. Volk HW, Schneider J, Dämmrich J, Döll W, Hörl M, Bruch H-P. Animal model for chronic-abscess-forming peritonitis: histology and microbiology. Eur Surg Res. 1990;22:347-55.

34. Rotstein OD. Role of fibrin deposition in the pathogenesis of intraabdominal infection. Eur J Clin Microbiol Infect Dis. 1992;11:1064-8.

35. Montravers P, Tashk P, Dinh AT. Unmet needs in the management of intra-abdominal infections. Expert Rev Anti-infe. 2017;15:839-50.

36. Zhu Y, Xu L, Liu W, Qi W, Cao Q, Zhou W. Safety and efficacy of exclusive enteral nutrition for percutaneously undrainable abdominal abscesses in Crohn's disease. Gastroent Res Pract. 2017;2017:1-8.

37. Cheng Y, Zhou S, Zhou R, Lu J, Wu S, Xiong X, et al. Abdominal drainage to prevent intra-peritoneal abscess after open appendectomy for complicated appendicitis. Cochrane Database Syst Rev. 2015. https://doi.org/10.1002/14651858.CD010 168.pub2.

38. Bhoir LN, Jagne NY, Murali D. Is using peritoneal drains in bowel surgeries beneficial? Int Surg J. 2017;4:650-5.

39. Podda M, Saverio SD, Davies RJ, Atzeni J, Balestra F, Virdis F, et al. Prophylactic intra-abdominal drainage following colorectal anastomoses. A systematic review and meta-analysis of randomized controlled trials. Am J Surg. 2019;219:164-74.

40. Puleo F, Mishra N, Hall J. Use of intra-abdominal drains. Clin Colon Rect Surg. 2013;26:174-7.

41. Bass GA, Seamon MJ, Schwab CW. A surgeon's history of the omentum: from omens to patches to immunity. J Trauma Acute Care. 2020;89:e161-6.

42. Horwood J, Akbar F, Maw A. Initial experience of laparostomy with immediate vacuum therapy in patients with severe peritonitis. Ann Royal Coll Surg Engl. 2009;91:681-7.

43. Perez D, Wildi S, Demartines N, Bramkamp M, Koehler C, Clavien P-A. Prospective evaluation of vacuum-assisted closure in abdominal compartment syndrome and severe abdominal sepsis. J Am Coll Surgeons. 2007;205:586-92.

44. Quyn AJ, Johnston C, Hall D, Chambers A, Arapova N, Ogston $\mathrm{S}$, et al. The open abdomen and temporary abdominal closure systems-historical evolution and systematic review. Colorectal Dis. 2012;14:e429-38.

45. D'Hondt M, D'Haeninck A, Dedrye L, Penninckx F, Aerts R. Can vacuum-assisted closure and instillation therapy (VACInstill@ therapy) play a role in the treatment of the infected open abdomen? Tech Coloproctol. 2011;15:75-7.

46. Kirkpatrick AW, Coccolini F, Ansaloni L, Roberts DJ, Tolonen $\mathrm{M}, \mathrm{McKee} \mathrm{JL}$, et al. Closed or open after source control laparotomy for severe complicated intra-abdominal sepsis (the COOL trial): study protocol for a randomized controlled trial. World J Emerg Surg. 2018;13:26.

47. Loftus TJ, Efron PA, Bala TM, Rosenthal MD, Croft CA, Walters MS, et al. The impact of standardized protocol implementation for surgical damage control and temporary abdominal closure after emergent laparotomy. J Trauma Acute Care. 2019;86(4):670-678.

48. Peerapornratana S, Manrique-Caballero CL, Gómez H, Kellum JA. Acute kidney injury from sepsis: current concepts, epidemiology, pathophysiology, prevention and treatment. Kidney Int. 2019;96:1083-99. 
49. Torer N, Yorganci K, Elker D, Sayek I. Prognostic factors of the mortality of postoperative intraabdominal infections. Infection. 2010;38:255-60.

50. Crouser ED. Sepsis-induced renal failure. Crit Care Med. 2018;46:658-60.

51. Vincent J-L, Nelson DR, Williams MD. Is worsening multiple organ failure the cause of death in patients with severe sepsis?*. Crit Care Med. 2011;39:1050-5.

52. Rosenthal M.D., Kamel A.Y., Rosenthal C.M., Brakenridge S., Croft C.A., Moore F.A. Chronic critical illness: Application of what we know. Nutr. Clin. Pract. 2018;33:39-45. https://doi. org/10.1002/ncp.10024

53. Maddux AB, Hiller TD, Overdier KH, Pyle LL, Douglas IS. Innate immune function and organ failure recovery in adults with sepsis. J Intensive Care Med. 2017;34:486-94.

54. Zhang Z, Ho KM, Gu H, Hong Y, Yu Y. Defining persistent critical illness based on growth trajectories in patients with sepsis. Crit Care. 2020;24:57.

55. Neto AS, Hemmes SN, Barbas CS, Beiderlinden M, FernandezBustamante A, Futier E, et al. Incidence of mortality and morbidity related to postoperative lung injury in patients who have undergone abdominal or thoracic surgery: a systematic review and meta-analysis. Lancet Respir Med. 2014;2:1007-15.

56. Choudhuri AH, Batra UB, Harisinghani P, Uppal R. Epidemiology of ventilator associated pneumonia (vap) in patients with abdominal sepsis. Intensive Care Med Exp. 2015;3:A280.

57. Meagher AD, Lind M, Senekjian L, Iwuchukwu C, Lynch JB, Cuschieri J, et al. Ventilator-associated events, not ventilatorassociated pneumonia, is associated with higher mortality in trauma patients. J Trauma Acute Care. 2019;87:307-14.

58. Kobayashi $\mathrm{H}$, Uchino S, Takinami M, Uezono S. The impact of ventilator-associated events in critically ill subjects with prolonged mechanical ventilation. Respir Care. 2017;62:1379-86.

59. Rimmelé T, Kellum JA. Clinical review: blood purification for sepsis. Crit Care. 2011;15:205.

60. Girardot T, Schneider A, Rimmelé T. Blood purification techniques for sepsis and septic AKI. Semin Nephrol. 2019;39:505-14.

61. Vinclair M, Broux C, Faure P, Brun J, Genty C, Jacquot C, et al. Duration of adrenal inhibition following a single dose of etomidate in critically ill patients. Intensive Care Med. 2008;34:714-9.

62. Cherfan AJ, Arabi YM, Al-Dorzi HM, Kenny LP. Advantages and disadvantages of etomidate use for intubation of patients with sepsis. Pharmacother J Hum Pharmacol Drug Ther. 2012;32:475-82.

63. Bruder EA, Ball IM, Ridi S, Pickett W, Hohl C. Single induction dose of etomidate versus other induction agents for endotracheal intubation in critically ill patients. Cochrane Database Syst Rev. 2015. https://doi.org/10.1002/14651858. CD010225.pub2.

64. Piper GL, Maerz LL, Schuster KM, Maung AA, Luckianow GM, Davis KA, et al. When the ICU is the operating room. J Trauma Acute Care. 2013;74:871-5.

65. Lane-Fall MB, Pascual JL, Massa S, Collard ML, Peifer HG, Taranti LJD, et al. Developing a standard handoff process for operating room-to-ICU transitions: multidisciplinary clinician perspectives from the handoffs and transitions in critical care (HATRICC) study. Jt Comm J Qual Patient Saf. 2018;44:514-25.

66. Lane-Fall MB, Pascual JL, Peifer HG, Taranti LJD, Collard ML, Jablonski J, et al. A partially structured postoperative handoff protocol improves communication in 2 mixed surgical intensive care units: findings from the handoffs and transitions in critical care (HATRICC) prospective cohort study. Ann Surg. 2020;271:484-93.
67. Rhodes A, Evans LE, Alhazzani W, Levy MM, Antonelli M, Ferrer R, et al. Surviving sepsis campaign: international guidelines for management of sepsis and septic shock: 2016. Crit Care Med. 2017;45:486-552.

68. Mazuski JE, Tessier JM, May AK, Sawyer RG, Nadler EP, Rosengart MR, et al. The surgical infection society revised guidelines on the management of intra-abdominal infection. Surg Infect. 2017;18:1-76.

69. Boucher BA, Wood GC, Swanson JM. Pharmacokinetic changes in critical illness. Crit Care Clin. 2006;22:255-71.

70. Roberts DJ, Hall RI. Drug absorption, distribution, metabolism and excretion considerations in critically ill adults. Expert Opin Drug Met. 2013;9:1-18.

71. Smith BS, Yogaratnam D, Levasseur-Franklin KE, Forni A, Fong J. Introduction to drug pharmacokinetics in the critically ill patient. Chest. 2012;141:1327-36.

72. Gross AS. Best practice in therapeutic drug monitoring: best practice in therapeutic drug monitoring. Br J Clin Pharmacol. 2001;52:5-9.

73. Abdul-Aziz MH, Alffenaar J-WC, Bassetti M, Bracht H, Dimopoulos G, Marriott D, et al. Antimicrobial therapeutic drug monitoring in critically ill adult patients: a position paper\#. Intensive Care Med. 2020;46:1127-53.

74. Mazuski JE, Solomkin JS. Intra-abdominal infections. Surg Clin N Am. 2009;89:421-37.

75. Hawser S, Hoban DJ, Badal RE, Bouchillon SK, Biedenbach D, Hackel M, et al. Epidemiology and antimicrobial susceptibility of Gram-negative aerobic bacteria causing intra-abdominal infections during 2010-2011. J Chemother. 2014;27:67-73.

76. Montravers P, Tubach F, Lescot T, Veber B, Esposito-Farèse $\mathrm{M}$, Seguin $\mathrm{P}$, et al. Short-course antibiotic therapy for critically ill patients treated for postoperative intra-abdominal infection: the DURAPOP randomised clinical trial. Intens Care Med. 2018;44:300-10.

77. Sawyer RG, Claridge JA, Nathens AB, Rotstein OD, Duane TM, Evans HL, et al. Trial of short-course antimicrobial therapy for intraabdominal infection. New Engl J Med. 2015;372:1996-2005.

78. Pepper DJ, Sun J, Rhee C, Welsh J, Powers JH, Danner RL, et al. Procalcitonin-guided antibiotic discontinuation and mortality in critically ill adults a systematic review and meta-analysis. Chest. 2019;155:1109-18.

79. Sager R, Kutz A, Mueller B, Schuetz P. Procalcitonin-guided diagnosis and antibiotic stewardship revisited. Bmc Med. 2017;15:15.

80. Taylor BE, McClave SA, Martindale RG, Warren MM, Johnson DR, Braunschweig C, et al. Guidelines for the provision and assessment of nutrition support therapy in the adult critically ill patient. Crit Care Med. 2016;44:390-438.

81. Stortz JA, Mira JC, Raymond SL, Loftus TJ, Ozrazgat-Baslanti $\mathrm{T}$, Wang $\mathrm{Z}$, et al. Benchmarking clinical outcomes and the immunocatabolic phenotype of chronic critical illness after sepsis in surgical intensive care unit patients. J Trauma Acute Care. 2018;84:342-9.

82. Singer P, Blaser AR, Berger MM, Alhazzani W, Calder PC, Casaer MP, et al. ESPEN guideline on clinical nutrition in the intensive care unit. Clin Nutr. 2019;38:48-79.

83. Rosenthal MD, Vanzant EL, Martindale RG, Moore FA. Evolving paradigms in the nutritional support of critically ill surgical patients. Curr Prob Surg. 2015;52:147-82.

84. Cheatham ML, Safcsak K, Brzezinski SJ, Lube MW. Nitrogen balance, protein loss, and the open abdomen*. Crit Care Med. 2007;35:127-31.

85. Hourigan LA, Hourigan L, Linfoot JA, Linfoot J, Chung $\mathrm{KK}$, Chung K, et al. Loss of protein, immunoglobulins, and 
electrolytes in exudates from negative pressure wound therapy. Nutr Clin Pract. 2010;25:510-6.

86. Herndon DN, Tompkins RG. Support of the metabolic response to burn injury. Lancet. 2004;363:1895-902.

87. Porro LJ, Herndon DN, Rodriguez NA, Jennings K, Klein GL, Mlcak RP, et al. Five-year outcomes after oxandrolone administration in severely burned children: a randomized clinical trial of safety and efficacy. J Am Coll Surgeons. 2012;214:489-502.

88. Herndon DN, Hart DW, Wolf SE, Chinkes DL, Wolfe RR. Reversal of catabolism by beta-blockade after severe burns. New Engl J Med. 2001;345:1223-9.

89. den Berghe GV. On the neuroendocrinopathy of critical illness. Perspectives for feeding and novel treatments. Am J Resp Crit Care. 2016;194:1337-48.

90. Stanculescu D, Larsson L, Bergquist J. Hypothesis: mechanisms that prevent recovery in prolonged ICU patients also underlie myalgic encephalomyelitis/chronic fatigue syndrome (ME/CFS). Front Med. 2021;8: 628029.

91. Takala J, Ruokonen E, Webster NR, Nielsen MS, Zandstra DF, Vundelinckx G, et al. Increased mortality associated with growth hormone treatment in critically ill adults. New Engl J Med. 1999;341:785-92.

92. Mathias B, Delmas AL, Ozrazgat-Baslanti T, Vanzant EL, Szpila BE, Mohr AM, et al. Human myeloid-derived suppressor cells are associated with chronic immune suppression after severe sepsis and sol; septic shock. Ann Surg. 2017;265:827-34.

93. Delphan M, Lin T, Liesenfeld DB, Nattenmüller J, Böhm JT, Gigic B, et al. Associations of branched-chain amino acids with parameters of energy balance and survival in colorectal cancer patients: results from the ColoCare study. Metabolomics. 2018;14:22.

94. Yu J, Liu L, Zhang Y, Wei J, Yang F. Effects of omega-3 fatty acids on patients undergoing surgery for gastrointestinal malignancy: a systematic review and meta-analysis. BMC Cancer. 2017; $17: 271$

95. Serhan CN, Levy BD. Resolvins in inflammation: emergence of the pro-resolving superfamily of mediators. J Clin Invest. 2018;128:2657-69.
96. Chiang N, Serhan CN. Specialized pro-resolving mediator network: an update on production and actions. Essays Biochem. 2020;64:443-62.

97. Network NH Lung, and Blood Institute PETAL Clinical Trials, Ginde AA, Brower RG, Caterino JM, Finck L, Banner-Goodspeed VM, et al. Early high-dose vitamin D3 for critically ill, vitamin D-deficient patients. New Engl J Med. 2019;381:2529-40.

98. Grootswagers P, Smeets E, de Groot L. A novel oral nutritional supplement improves gait speed in Dutch older adults with (risk of) undernutrition. Clin Nutr. 2018;37:S6.

99. Verreijen AM, de Wilde J, Engberink MF, Swinkels S, Verlaan S, Weijs PJ. OP007 a high whey protein, leucine enriched supplement preserves muscle mass during intentional weight loss in obese older adults: a double blind randomized controlled trial. Clin Nutr. 2013;32:S3.

100. McPeake J, Mikkelsen ME. The evolution of post intensive care syndrome. Crit Care Med. 2018;46:1551-2.

101. Zante B, Camenisch SA, Schefold JC. Interventions in postintensive care syndrome-family: a systematic literature review. Crit Care Med. 2020;48(9):e835-40 (Publish Ahead of Print).

102. Kok N, van Gurp J, Teerenstra S, van der Hoeven H, Fuchs M, Hoedemaekers C, et al. Coronavirus disease 2019 immediately increases burnout symptoms in ICU professionals: a longitudinal cohort study*. Crit Care Med. 2021;49:419-27.

103. Moss M, Good VS, Gozal D, Kleinpell R, Sessler CN. An official critical care societies collaborative statement: burnout syndrome in critical care health care professionals: a call for action. Am J Crit Care. 2016;25:368-76.

104. Duffy CC, Bass GA, Fitzpatrick G, Doherty EM. What can we learn from the past? Pandemic health care workers' fears, concerns, and needs: a review. J Patient Saf. 2020. https://doi.org/ 10.1097/PTS.0000000000000803 (Publish Ahead of Print). 